\title{
Proposed ZAH_BAU filter for RGB color image enhancement
}

\author{
Ashraf Abdel-Karim Abu-Ein ${ }^{1}$, Ziad A. Alqadi ${ }^{2}$ and Hazem (Moh'd Said) Hatamleh ${ }^{3}$ \\ ${ }^{1}$ Computer Engineering and Computer Networks Department, Al-Balqa' Applied University \\ Amman, 15008 Amman 11134 Jordan, Jordan \\ ${ }^{2}$ Computer Engineering and Computer Networks Department, Al-Balqa' Applied University \\ Amman, 15008 Amman 11134 Jordan, Jordan \\ ${ }^{3}$ Applied Science Department, Al-Balqa' Applied University \\ Ajloun, Jordan
}

\begin{abstract}
Image contrast enhancement is an important step in almost every digital image preprocessing phase. The objective of image enhancement is to increase the visual perception of the image so that it is more suitable for human viewers and readers or machine vision applications. In this paper a new ZAH_BAU filter will be proposed, this filter can be used to control the brightness of RGB color image, and control individual channel in color image. The filter can be used also for different applications such as certain color removal and color image enhancement.
\end{abstract}

Keywords: RGB image, HSV image, ZAH_BAU filter, color removing.

\section{Introduction}

RGB color image is a 3 dimensional matrix [1], [2], [3], and it is an additive color model. It means that different proportions of Red, Blue and Green light can be used to produce color. The RGB color model was created specifically for display purposes (display screens, projectors etc).

HSV color system is based on the Hue shift, Saturation and Value. Unlike the RGB color system, which has to do with "implementation details" regarding the way RGB displays color, HSV has to do with the "actual color" components. Another way to say this would be RGB is the way computers treats color, and HSV try to capture the components of the way we humans perceive color. The main reason to work on the HSV version of an image is because using Hue component makes the algorithms less sensitive (if not invariant) to lighting variations. Because HSL and HSV are simple transformations of devicedependent RGB models, the physical colors they define depend on the colors of the red, green, and blue primaries of the device or of the particular RGB space[4],[5],[6], table 1 shows some RGB values and there equivalents in HSV model.

To convert the R,G,B of the color image values are divided by 255 to change the range from $0 . .255$ to $0 . .1$ we can apply the following formulas:

$\mathrm{R}^{\prime}=\mathrm{R} / 255$

$\mathrm{G}^{\prime}=\mathrm{G} / 255$

$\mathrm{B}^{\prime}=\mathrm{B} / 255$

$\mathrm{Cmax}=\max \left(\mathrm{R}^{\prime}, \mathrm{G}^{\prime}, \mathrm{B}^{\prime}\right)$

$\mathrm{Cmin}=\min \left(\mathrm{R}^{\prime}, \mathrm{G}^{\prime}, \mathrm{B}^{\prime}\right)$

$\Delta=\mathrm{Cmax}-\mathrm{Cmin}$

Hue calculation:

$H=\left\{\begin{array}{cc}0^{\circ} & \Delta=0 \\ 60^{\circ} \times\left(\frac{G^{\prime}-B^{\prime}}{\Delta} \bmod 6\right) & , C_{\max }=P^{\prime} \\ 60^{\circ} \times\left(\frac{B^{\prime}-R^{\prime}}{\Delta}+2\right) & , C_{\max }=G^{\prime} \\ 60^{\circ} \times\left(\frac{R^{\prime}-G^{\prime}}{\Delta}+4\right) & , C_{\max }=B^{\prime}\end{array}\right.$

Saturation calculation:

$S=\left\{\begin{array}{cl}0 & , C_{\max }=0 \\ \frac{\Delta}{C_{\max }} & , C_{\max } \neq 0\end{array}\right.$

Value calculation:

$\mathrm{V}=\mathrm{Cmax}$

Table 1: RGB values and there equivalents in HSV model

\begin{tabular}{|c|c|c|c|}
\hline Color & $\begin{array}{l}\text { Color } \\
\text { name }\end{array}$ & $(\mathrm{R}, \mathrm{G}, \mathrm{B})$ & $(\mathrm{H}, \mathrm{S}, \mathrm{V})$ \\
\hline & Black & $(0,0,0)$ & $\left(0^{\circ}, 0 \%, 0 \%\right)$ \\
\hline White & $(255,255,255)$ & $\left(0^{\circ}, 0 \%, 100 \%\right)$ \\
\hline Red & $(255,0,0)$ & $\left(0^{\circ}, 100 \%, 100 \%\right)$ \\
\hline & Lime & $(0,255,0)$ & $\left(120^{\circ}, 100 \%, 100 \%\right)$ \\
\hline
\end{tabular}




\begin{tabular}{|c|c|c|}
\hline Blue & $(0,0,255)$ & $\left(240^{\circ}, 100 \%, 100 \%\right)$ \\
\hline Yellow & $(255,255,0)$ & $\left(60^{\circ}, 100 \%, 100 \%\right)$ \\
\hline Cyan & $(0,255,255)$ & $\left(180^{\circ}, 100 \%, 100 \%\right)$ \\
\hline Magenta & $(255,0,255)$ & $\left(300^{\circ}, 100 \%, 100 \%\right)$ \\
\hline Silver & $(192,192,192)$ & $\left(0^{\circ}, 0 \%, 75 \%\right)$ \\
\hline Gray & $(128,128,128)$ & $\left(0^{\circ}, 0 \%, 50 \%\right)$ \\
\hline Maroon & $(128,0,0)$ & $\left(0^{\circ}, 100 \%, 50 \%\right)$ \\
\hline Olive & $(128,128,0)$ & $\left(60^{\circ}, 100 \%, 50 \%\right)$ \\
\hline Green & $(0,128,0)$ & $\left(120^{\circ}, 100 \%, 50 \%\right)$ \\
\hline Purple & $(128,0,128)$ & $\left(300^{\circ}, 100 \%, 50 \%\right)$ \\
\hline Teal & $(0,128,128)$ & $\left(180^{\circ}, 100 \%, 50 \%\right)$ \\
\hline Navy & $(0,0,128)$ & $\left(240^{\circ}, 100 \%, 50 \%\right)$ \\
\hline
\end{tabular}

\section{Related works}

Image contrast enhancement is an important step in almost every digital image preprocessing phase. The objective of image enhancement is to increase the visual perception of the image so that it is more suitable for human viewers and readers or machine vision applications [4], [5]. One of the mostly used methods to examine the clearance of the gray image is to represent the image by a histogram [6]. The histogram in Fig. 1 represents the pixels in the image with a vertical bar for each of the 256 brightness levels from 0 to 255 . The height of the bar indicates the relative number of pixels for a given brightness level. Brightness level 0 is at the far left side of the histogram, and level 255 is at the far right hand side. Shades of gray represented by different brightness levels range in between [7].

Because we have a limited amount of space for the histogram display, each vertical bar that represents an individual brightness level will be very narrow. The bars will be crowded touching each other without any space in between, if we do, in fact, we will have pixels for each possible level in the image. There are 256 different brightness levels to work with in an 8-bit image. Each level is represented by a vertical bar in the histogram's bar graph, but because there are so many to be displayed in such a limited space, individual bars cannot be differentiated because they all run together. By examining the histogram, we can tell if data has been lost, or if the brightness range of the image does not match the available dynamic range we have to work with[8], [9].

\section{Unused Dynamic Range $=$ Tones}

Not Used Since we have a limited range of tones to work with in a digitized image (usually 256 in each channel), one of our main goals is to make sure we closely match the brightness range of the image to the available dynamic range of the tones we have to work with. Doing so optimizes the tonal data and maximizes the contrast of the image [10].

\section{Clipped Data $=$ Data Lost}

If we overexpose a slide film, details will not be recorded in the important highlights. On the film where the highlights should be, the film will literally be transparent. There will be no data recorded there. If this happens, there is no way to restore these lost details later. Likewise, if we underexpose an image, no details will be recorded in critical shadow areas of the image [11], [12]. If such detail are not recorded when the exposure is made, there is no way to put it in later. Similar situations can occur when scanning an image. If some details present in the original film image are not recorded in the scan, then there is no recovery possible later unless it is re-scanned. Details in the shadows and highlights of an image can be lost by the scanner setting the wrong exposure for the clipped shadow detail (CSD) in the pre-scan, or by the scanner operator incorrectly setting the black and white points after the prescan, or by incorrectly adjusting the brightness or contrast of the scan.

Removing unwanted parts or colors [13], leads to image enhancement but we have still need to control the parts or colors in RGB image to be removed, Here an example of how we can control the process of color removing (Figure 1) by processing the following mat lab code: myrgb $=\operatorname{rand}(50,50,3)$; imagesc $($ myrgb $)$, figure floatNorm $=255 ; \operatorname{tad}=\operatorname{repmat}(4,[50,50,3])$; target $=\operatorname{repmat}([105,112,175], 50 * 50,1)$; target $=$ reshape $(\operatorname{target},[50,50,3])$; threshold $=($ target + tad $) /$ floatNorm; myFilt $=$ abs $($ myrgb $-1 / 2)<=$ threshold; $\operatorname{myrgb}($ myFilt $)=0 ;$ imagesc $($ myrgb $)$ 


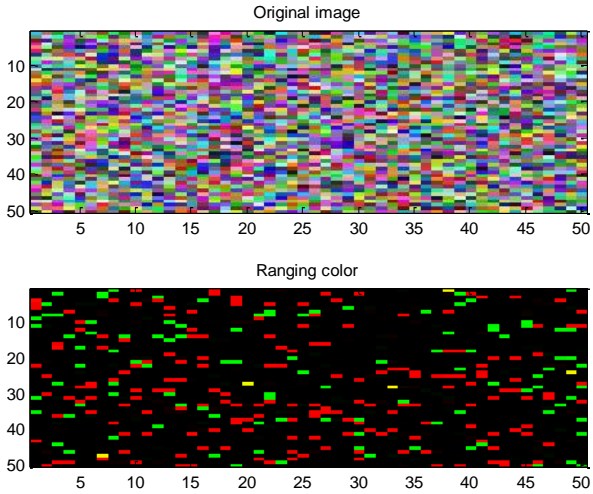

Figure 1: Color removing and enhancement

\section{The proposed ZAH_BAU filter}

The proposed ZAH_BAU filter (stands for Ziad Ashraf Hazem Al-Balqa' Applied University) can be implemented applying the following steps:

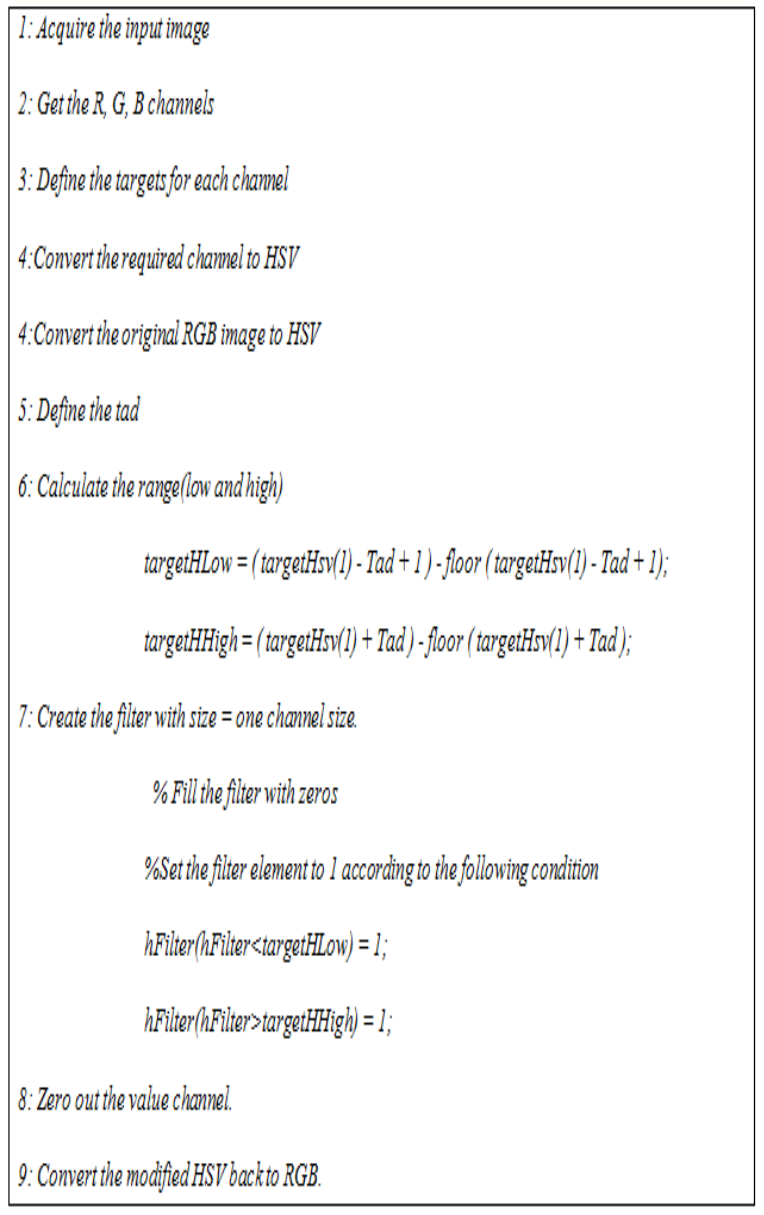

Here we can control the tad, the rang (low and high values, the channel to be enhanced, thus giving more flexibility to the user to apply the filter for deferent application.

\section{ZAH_BAU filter implementation}

The following matlab code was written to implement the proposed algorithm close all, clear all, clc

$\%$ step 1: Acqiure the input image

$\mathrm{a}=$ imread('peppers.png');

raw_frame $=\mathrm{a}$;

imshow(a), title 'Original image'

figure

\%step 2: Get the R, G, B channels

redChannel $=\mathrm{a}(:,:, 1)$;

greenChannel $=\mathrm{a}(:,,:, 2)$;

blueChannel $=\mathrm{a}(:,:, 3)$;

[redCount grayCount] = imhist(redChannel);

[greenCount grayCount] = imhist $($ greenChannel $)$;

[blueCount grayCount] = imhist(blueChannel);

plot(grayCount,redCount,grayCount,greenCount,grayCoun t,blueCount)

legend('Original Red','Original Green','Original Blue')

tic

\%step 3: Define the targets for each channel

$\operatorname{targetRgb}(1,1,1)=105$;

$\operatorname{target} \operatorname{Rgb}(1,1,2)=112$

$\operatorname{targetRgb}(1,1,3)=175$

\%step 4:Convert the required channel to HSV

targetHsv = rgb2hsv (targetRgb);

\% step 5:Convert the original RGB image to HSV

frameHsv = rgb2hsv(raw_frame);

frameSize = size (raw_frame);

\%step 6: Define the tad

$\mathrm{hTad}=0.05$

\%Step 7: Calculate the range(low and high)

targetHLow $=(\operatorname{targetHsv}(1)-\mathrm{hTad}+1)-$ floor (targetHsv(1) - hTad + 1);

targetHHigh $=(\operatorname{targetHsv}(1)+\mathrm{hTad})-$ floor $(\operatorname{targetHsv}(1)$

+ hTad );

\%Step 8: Create the filter with size $=$ one channel size.

$\%$ Fill the filter with zeros

hFilter = zeros(frameSize(1), frameSize(2));

$\%$ Set the filter element to 1 according to the following condition

hFilter(hFilter $<$ targetHLow $)=1$;

hFilter(hFilter $>$ targetHHigh $)=1$;

\%Step 9: Zero out the value channel.

frameV = frameHsv(:,:,3);

frameV(hFilter $)=0$;

frameHsv(:,:,3) = frameV;

\% Step 10: Convert the modified HSV back to RGB. 
frameFiltered $=$ hsv2rgb(frameHsv);

toc

figure,imshow(frameFiltered), title 'Filtered image'

figure

redChannel $=$ frameFiltered $(:,:, 1)$;

greenChannel $=$ frameFiltered $(:,:, 2)$;

blueChannel $=$ frameFiltered $(:,:, 3)$;

[redCount grayCount $]=$ imhist( redChannel);

[greenCount grayCount] = imhist $($ greenChannel $)$;

[blueCount grayCount] = imhist(blueChannel);

plot(grayCount,redCount, grayCount,greenCount,grayCoun

t, blueCount)

legend ('Filtered Red','Filtered Green','Filtered Blue')

Several RGB images with deferent sizes were treated using the proposed algorithm and the results of implementation satisfied the needed process of RGB color image enhancement, figure 2 to 5 shows some experimental results.

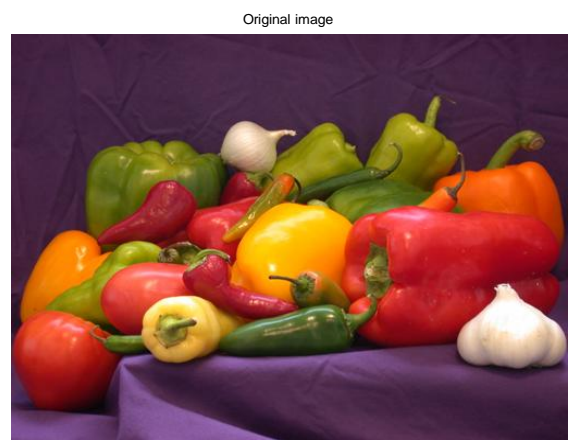

FIGURE 2: ORIGINAL INPUT IMAGE

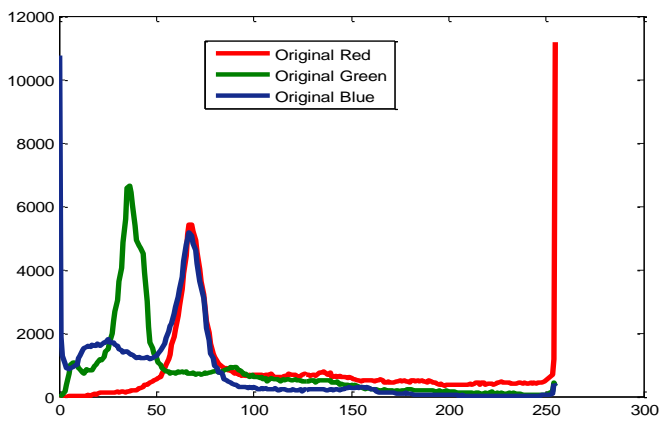

FIGURE 3: ORIGINAL IMAGE CHANNELS VALUES

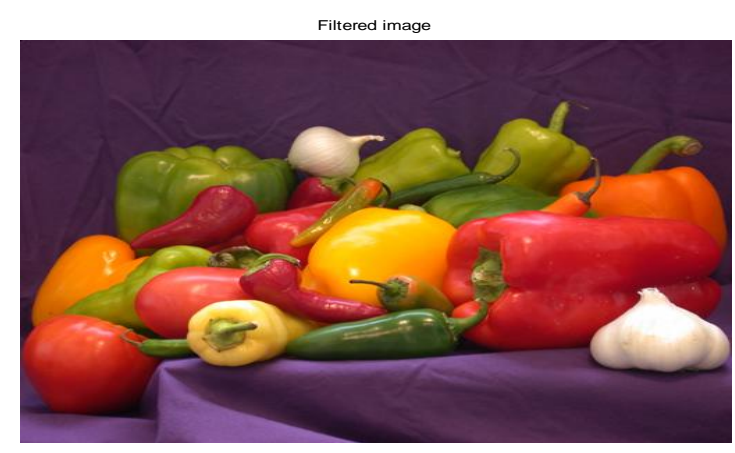

FigURE 3: FiLTERED IMAGE

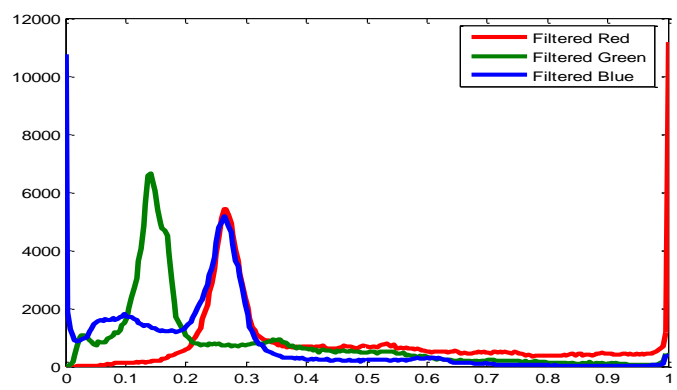

FIGURE 3: FILTERED IMAGE CHANNELS VALUES

The execution time of the filter does not exceed 0.156000 seconds.

\section{Conclusions}

Taking the results of implementation in consideration we can conclude the following facts:

- The proposed filter suits gray and color image enhancement.

- The proposed filter can be used to remove unwanted colors within RGB image or unwanted colors within a color channel.

- The parameters of the proposed filter (Low, High, range, Tad and channels) are controllable by the user.

- The proposed filter is flexible and efficient.

\section{References}

[1] Jamil Al Azzeh, Ziad A. Alqadi, Hussein Alhatamleh, 2016. Mohammad Khalil Abuzalata, Creating a Color Map to be used to Convert a Gray Image to Color Image, International Journal of Computer Applications (0975 - 8887) Volume 153 - No 2.

[2]: Majed O Al-Dwairi-Ziad A Alqadi - Amjad A Abujazar · Rushdi Abu Zneit, Optimized True-Color Image Processing, WAS journal: 8(10), 1175-1182, 2011.

[3]: Haitham Alasha'ary, Abdullah Al-Hasanat, Khaled Matrouk, Ziad Al-Qadi, Hasan Al-Shalabi, 2014. A Novel Digital 
Filter for Enhancing Dark Gray Images, European Journal of Scientific Research ISSN 1450-216X / 1450-202X Vol.122 No.1, pp.99-106.

[4] Barten P. G., 1999. "Contrast Sensitivity of the Human Eye and its Effects on Image Quality", SPIE - The International Society for Optical Engineering, P.O. Box 10 Bellingham Washington 98227-0010. ISBN 0-8194-3496-5.

[5] Fattal R., Lischinski D., and Werman M., 2002. "Gradient Domain High Dynamic Range Compression", ACM Transactions on Graphics, Proceedings of ACM Siggraph 21(3), pp. 249-256.

[6] Hanmandlu M., Jha D., and Sharma R., 2000. "Color Image Enhancement by Fuzzy Intensification", Proceedings of the International Conference on Pattern Recognition.

[7] Hanmandlu M., Jha D., and Sharma R., 2001. "Localized Contrast Enhancement of Color Images Using Clustering", Proceedings of the IEEE International Conference on Information Technology: Coding and Computing (ITCC).

[8]: Waheeb Abu Ulbeh, Akram Moustafa, Ziad A Alqadi, Gray image reconstruction, European Journal of Scientific Research, vol.27-2,pp 167-173,2009.

[9] Akram A Moustafa, Ziad A Alqadi, 2009. A Practical Approach of Selecting the Edge Detector Parameters to Achieve a Good Edge Map of the Gray Image, Journal of Computer Science 5 (5): 355-362, 2009 ISSN 1549-3636.

\section{Authors' information}

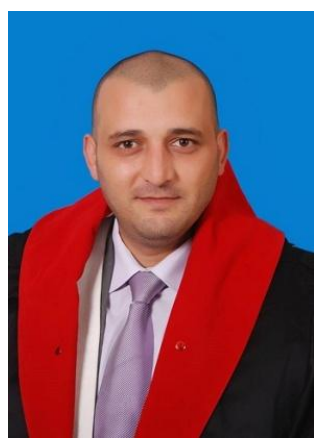

Dr. Ashraf Abdel-Karim Abu-Ein was born in Irbid, Jordan in 1979, he received his Ph.D. Degree in 2007 from the "National Technical University of Ukraine". $\mathrm{He}$ is currently Associate Professor of computer engineering and computer networks department of $\mathrm{Al}$ Balqa' University. His current research interest is including computer networks, image processing and computer graphics and digital image processing. $\mathrm{He}$ is the author and coauthor more than 20 paper in international and more than 10 paper in

conference proceedings.

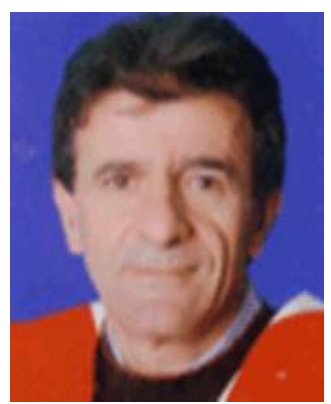

Prof. Ziad Alqadi he received the M.Sc. and PH.D degrees in 1986 from National Technical University of Ukraine". He is currently full Professor of computer engineering and computer networks department of Al-Balqa' University. His current research interest is including digital image processing, Algorithms, image processing and computer graphics.

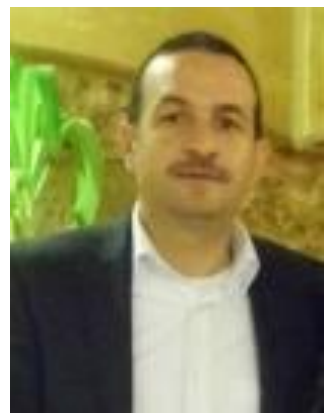

Dr. Hazem (Moh'd Said) abdel majid Hatamleh was born in irbid, Jordan in 1973, he received he M.Sc. and PH.D degrees in 2007 from the National Technical University of Ukraine". He is currently Associate Professor of Applied science department of Al-Balqa' University. His current research interest is including computer networks, wireless networks, and image processing and computer graphics. 